\begin{tabular}{|c|c|c|c|}
\hline Eiszeitalter u. Gegenwart & 41 & $\begin{array}{c}107-118 \\
3 \text { fig., 3 tab. }\end{array}$ & Hannover 1991 \\
\hline
\end{tabular}

\title{
La Téphra de Bag: une retombée volcanique à large dispersion dans le loess pléistocène d'Europe centrale
}

\author{
Etienne Juvigné, Elisabeth Horvāth \& Gyula Gábris *)
}

\begin{abstract}
Résumé: Plusieurs aspects d'une couche de téphra présente dans sept coupes de loess de Hongrie et de Tchécoslovaquie ont été étudiés: la granulométrie, l'association de minéraux mafiques et la composition chimique d'échantillons globaux ainsi que de clinopyroxènes. La téphra a été reconnue identique dans tous les gîtes étudiés, et le nom de "Téphra de Bag" lui a été donné. Cette téphra permet de proposer une corrélation nouvelle entre les coupes de loess concernées. La retombée volcanique a eu lieu pendant ou avant la cinquième dernière glaciation. En raison de la position très éloignée des champs volcaniques pleistocènes les plus proches (plus de $500 \mathrm{~km}$ ), la Téphra de Bag s'impose comme un marqueur stratigraphique à très large dispersion en Europe Centrale. Les données actuellement disponibles permettent de penser que le champ volcanique d'origine devrait être l'Eifel orientale (Allemagne) plutôt que l'Apennin central (Italie) ou les Carpates orientales (Roumanie). La corrélation avec un volcan du Massif Central français ou de la mer Egée est exclue.
\end{abstract}

[Die „Tephra von Bag”: ein weitverbreiteter vulkanischer Leithorizont im pleitozänen Löß Zentraleuropas]

Kurzfassung: Mehrere Eigenschaften (Korngröße, Mineralspektrum, chemische Zusammensetzung von Gesamtproben und von Klinopyroxenen) einer Tephralage, die in sieben Lößprofilen von Ungarn und der Tschechoslovakei vorkommt, wurden untersucht. Die Tephra ist identisch in allen Lokalitäten, und der Name „Tephra von Bag” wurde gewählt. Diese Tephra erlaubt eine neue Korrelation der untersuchten Lößprofile. Die Ablagerung der Tephra hat während oder vor der fünftletzten Eiszeit stattgefunden.

*) Adresses des auteurs: E. JuvignÉ, Chercheur Qualifié au F.N.R.S., Université de Liège, Laboratoire de Géomorphologie et de Géologie du Quaternaire, Place du XX Août, 4000 -Liège, Belgique.

E. HorvāTh, Assistante, et G. GĀBRIS, Professeur, Eötvös Loránd University, Département de Géographie Physique, Kun Béla Tér 2, 1083 - Budapest, Hongrie.
$\mathrm{Da}$ sich die nächsten vulkanischen pleistozänen Felder in einer Entfernung von mehr als $500 \mathrm{~km}$ befanden, kann die Tephra von Bag als weitverbreiteter Leithorizont in Zentraleuropa angesehen werden. Die zur Zeit verfügbaren Angaben erlauben die Schlußfolgerungen, daß der fördernde Vulkan sich eher in der Osteifel (Deutschland) als in dem Apennin (Italien) oder in den Karpaten (Rumänien) befindet. Die Korrelation mit einem Vulkan vom Zentral-Massiv (Frankreich) oder vom Agäischen Meer (Griechenland) wird ausgeschlossen.

[The "Bag Tephra": A Widespread Volcanic Stratigraphical Marker in the Pleistocene Loess of Central Europe]

Abstract: Several aspects of a tephra layer present in seven loess sections in Hungary and in Czekoslavakia were investigated: grain-size, mafic mineral suites, and chemical composition of bulk samples as well as clinopyroxenes. The tephra is identical in all localities, and the name "Bag Tephra" was given to it. This tephra allows us to propose a new stratigraphical correlation of the investigated loess sections. The tephra-fall occurred during or before the last 5 th glaciation. Since the closest pleistocene volcanic fields are situated very far from the investigated localities (more than $500 \mathrm{~km}$ ), the Bag Tephra can be used as a widespread stratigraphical marker in Central Europe. The currently available data show that the relevant volcano should be located in the East Eifel volcanic field (Germany), rather than in the Apennin (Italy) or in the East Carpathian Mountains (Romania). The correlation with any volcano in the French Central Massif or in the Agean See (Greece) is excluded.

\section{Introduction}

Une couche de téphra a été trouvée dans plusieurs coupes de loess en Hongrie par KrIVAN (1958) et par KRIVAN \& Rozsavolgyi (1964). Le moment de la retombée a été situé pendant la glaciation Riss sur la base de l'interprétation des données lithostratigraphiques des coupes contenant la téphra. Une analyse chimique globale d'une couche de téphra et la pré- 
sence systématique d'amphibole et de biotite dans les retombées ont permis d'attribuer la téphra au volcanisme andésitique des Carpates orientales.

VASKOVSKY \& KAROLUSOVA (1969) ont signalé la présence d'une couche de téphra dans une coupe de loess à Komjatice (Tchécoslovaquie) sous trois sols bruns forestiers. Le minéral mafique dominant en est l'augite, et cette téphra a été rapportée par les auteurs à un volcan basaltique indéterminé.

Les compositions des téphras rapportées ci-dessus impliquent l'absence de corrélation entre les retombées signalées d'une part en Hongrie et d'autre part en Tchécoslovaquie: celle de Hongrie est andésitique à hornblende, et celle de Tchécoslovaquie basaltique à augite.

Dans le présent article, nous montrons qu'il existe une téphra identique dans toutes les coupes étudiées antérieurement ainsi que dans la coupe de Pásztó (Fig. 1).

\section{La téphra dans les gîtes étudiés}

Sept coupes de loess réparties de la Slovaquie jusque dans le Sud de la Hongrie, ont été étudiées, elles sont localisées sur la figure 1 .

La couche de téphra se trouve suivant les coupes, sous 1 à 5 sols fossiles. Elle s'est mise en place pendant une phase de sédimentation loessique sous climat périglaciaire.

Il existe une différence de faciès entre les couches de téphra du sud de la Hongrie (Dunaszekcsö, Paks, Sióagárd) et les autres (Pásztó, Bag, Hévizgyörk, Komjatice). Les premières sont de couleur jaune à brun, tandis que les autres sont grises.

L'examen en laboratoire a montré que la téphra de chaque gîte est contaminée par le loess qui la jouxte et aussi par du calcaire secondaire.
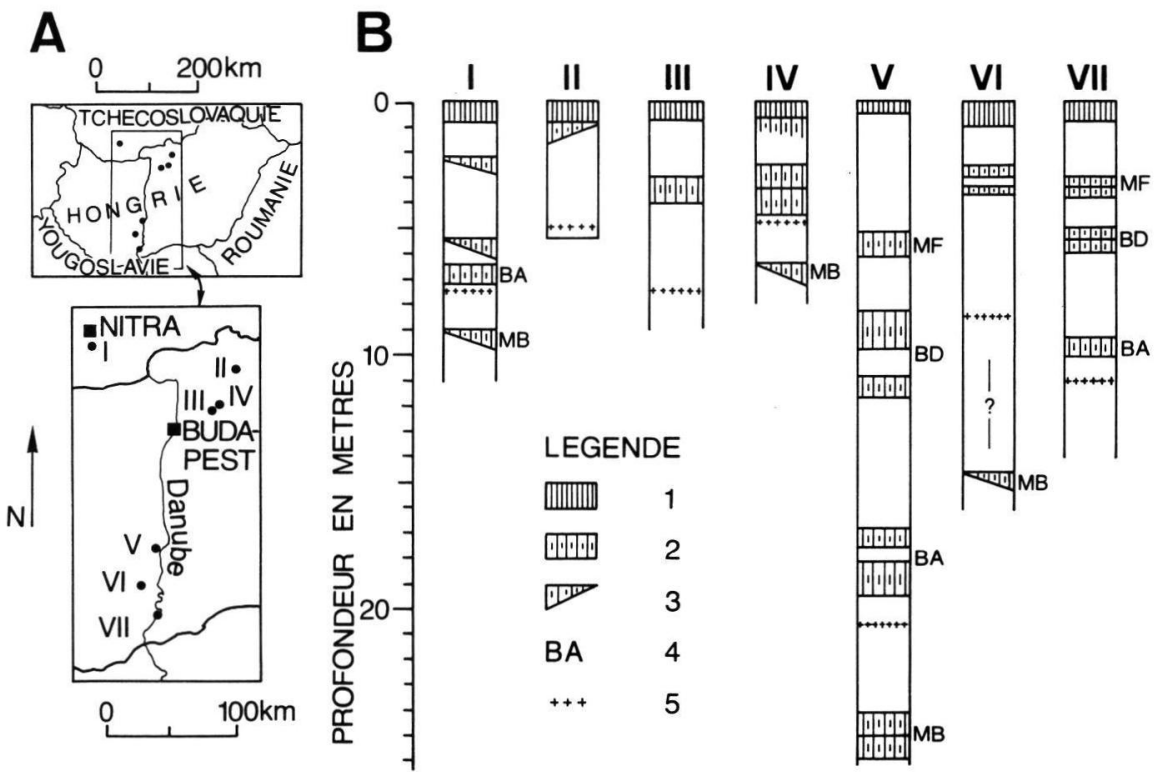

Figure 1: Localisation des gîtes étudiés (1 A) et position stratigraphique de la téphra dans chacun des profils de loess (1 B).

Légende. - 1A: I, Komjatice, ancienne briqueterie; II, Pásztó, site d'habitations tziganes;

III, Bag, ancienne briqueterie Csintovany à l'endroit de l'aire de repos nord sur l'autoroute;

IV, Hévizgyörk, coupe en face de la gare; V, Paks, briqueterie; VI, Sióagárd, versant d'érosion latérale de la Sió près des caves à vin; VII, Dunaszekcsö, escarpement du plateau loessique longeant la rue principale.

1B: 1, sol actuel; 2, sol fossile observé au droit de la téphra; 3, sol fossile observé latéralement par rapport à la séquence contenant la téphra; 4, connotations des sols selon Pécsi (1979); 5, couche de téphra.

Remarque. - Les sols fossiles sans connotation n'ont fait l'objet d'aucune interprétation chronostratigraphique publiée.

En raison des lacunes stratigraphiques importantes qui existent dans les coupes étudiées (voir plus loin),

il ne nous est pas possible d'établir des corrélations entre ces sols et ceux des coupes interprétées antérieurement. 


\section{Les minéraux mafiques}

\subsection{Observations au microscope polarisant}

Les minéraux denses ont été extraits de la fraction supérieure à $63 \mu \mathrm{m}$ de chaque échantillon. Le choix de cette limite granulométrique permet d'éliminer la plus grande part des minéraux des loess qui ont pénétré la téphra, et fournit ainsi des concentrés de minéraux volcaniques. Cette séparation a été réalisée de la détermination sont présentés dans le tableau 1.

Dans l'ensemble, les clinopyroxènes constituent plus de $94 \%$ de l'association des minéraux denses d'origine volcanique dans chacune des coupes. Ils sont verts, peu pléochrö̈ques et se présentent sous forme de grains idiomorphes, de fragments de prismes ou d'esquilles; les faciès d'altération sont rares.

Tableau 1: Associations de minéraux denses de la couche de téphra dans les sept gîtes étudíes.

Les parts des minéraux volcaniques sont emprimées en pour-cent: celles des minéraux non volcaniques (N.V.) en nombre de grains pour 100 minéraux volcaniques. par centrifugation dans le bromoforme. Les résultats

\subsection{Analyses de clinopyroxènes par microsonde}

Une dizaine de clinopyroxènes de chaque localité ont été analysés par microsonde et déterminés suivant la classification de MORIMOTO (1988). Les résultats sont représentés dans le tableau 2.

Tableau 2: Composition chimique des clinopyroxènes des sept gites étudiés (Les numéros I à VII correspondent à ceux des localités de la figure 1 .

\begin{tabular}{|c|c|c|c|c|c|c|c|}
\hline & I & II & III & IV & $\mathbf{V}$ & VI & VII \\
\hline $\mathrm{SiO}_{2}$ & 47,32 & 48,72 & 47,05 & 48,53 & 46,50 & 47,86 & 47,99 \\
\hline $\mathrm{TiO} 2$ & 1,09 & 0,89 & 1,10 & 0,93 & 1,09 & 0,98 & 0,95 \\
\hline $\mathrm{Al} 2 \mathrm{O} 3$ & 5,92 & 5,23 & 6,51 & 5,17 & 6,7 & 5,50 & 5,94 \\
\hline $\mathrm{Cr} 2 \mathrm{O} 3$ & 0,01 & 0,09 & 0,03 & 0,08 & 0,01 & 0,02 & 0,07 \\
\hline $\mathrm{FeOt}$ & 8,03 & 7,71 & 8,07 & 6,54 & 10,19 & 6,97 & 7,69 \\
\hline $\mathrm{MnO}$ & 0,26 & 0,22 & 0,11 & 0,15 & 0,27 & 0,12 & 0,16 \\
\hline $\mathrm{MgO}$ & 12,02 & 12,57 & 12,34 & 13,73 & 10,41 & 13,22 & 12,19 \\
\hline $\mathrm{CaO}$ & 24,13 & 23,58 & 24,22 & 24,13 & 23,6 & 24,45 & 23,74 \\
\hline $\mathrm{Na} 2 \mathrm{O}$ & 0,29 & 0,31 & 0,16 & 0,20 & 0,37 & 0,19 & 0,24 \\
\hline $\mathrm{K} 2 \mathrm{O}$ & 0,02 & 0,01 & 0,01 & 0,01 & 0,01 & 0,01 & 0,01 \\
\hline Total & 99,09 & 99,33 & 99,61 & 99,48 & 99,14 & 99,32 & 98,98 \\
\hline wo & 50,7 & 49,7 & 50,4 & 49,5 & 50,7 & 50,3 & 50,5 \\
\hline EN & 35,1 & 36,8 & 35,7 & 39,2 & 31,1 & 37,7 & 36,0 \\
\hline FS & 14 & 13,3 & 13,8 & 22,1 & 18,0 & 11,9 & 27,0 \\
\hline
\end{tabular}

Remarque. - Un tableau des résultats détaillés des analyses peut être obtenu sur demande à l'adresse de E. JuviGNÉ.

Tous les clinopyroxènes analysés appartiennent au "Quad-group". Dans le quadrilatère $\mathrm{Ca}-\mathrm{Mg}-\mathrm{Fe}$, ils sont très calciques et distribués en une série continue de part et d'autre de la limite des champs du diopside et des "pyroxènes inhabituels" (Fig. 2B/D/E) avec dans chaque cas $8<[100 * \mathrm{Fe} / \mathrm{Fe}+\mathrm{Mg}]<48$. Aucune différence fondamentale ne se dégage d'un gîte à l'autre. De plus, pratiquement tous les clinopyroxènes sont alumineux $\left(\mathrm{Al}^{3+}>0.1\right.$ dans la formule structurale). La majorité d'entre eux pourraient aussi être ferriques $\left(\mathrm{Fe}^{3+}>0.1\right.$ dans la formule structurale), mais à ce sujet, il faut tenir compte de ce que n'est pas possible de distinguer au microscope ceux de ces minéraux qui pourraient appartenir à l'un ou à l'autre de ces matériaux; ils ont été comptés avec les minéraux non volcaniques (Tab. 1: N.V.). le rapport $\mathrm{Fe}^{2+} / \mathrm{Fe}^{3+}$ n'est pas réel; il provient du fractionnement théorique du fer total suivant la méthode de HAMM \& VIETEN (1971).

En conclusion, les clinopyroxènes des sept gîtes étudiés appartiennent à une même population, ce qui constitue un argument en faveur de l'unicité de la retombée des sept gîtes étudiés.

\section{Granulométrie} stent aussi bien dans la téphra que dans les loess, et il

Les amphiboles ne représentent que quelques pourcent de l'association. Il existe dans les loess des parts ont pénétré la téphra. Inversement, il existe dans la téphra jusqu'à 3,8\% d'amphiboles brunes, un peu de sphène et d'olivine que l'on ne trouve qu'à l'état de traces infimes dans le loess; ces trois minéraux on donc été attribués à la téphra.

La biotite et, dans une moindre mesure, l'apatite exiUne étude granulométrique globale ne peut fournir de résultats représentatifs des mécanismes de mise en place de la téphra. En effet le verre volcanique a été 
S】
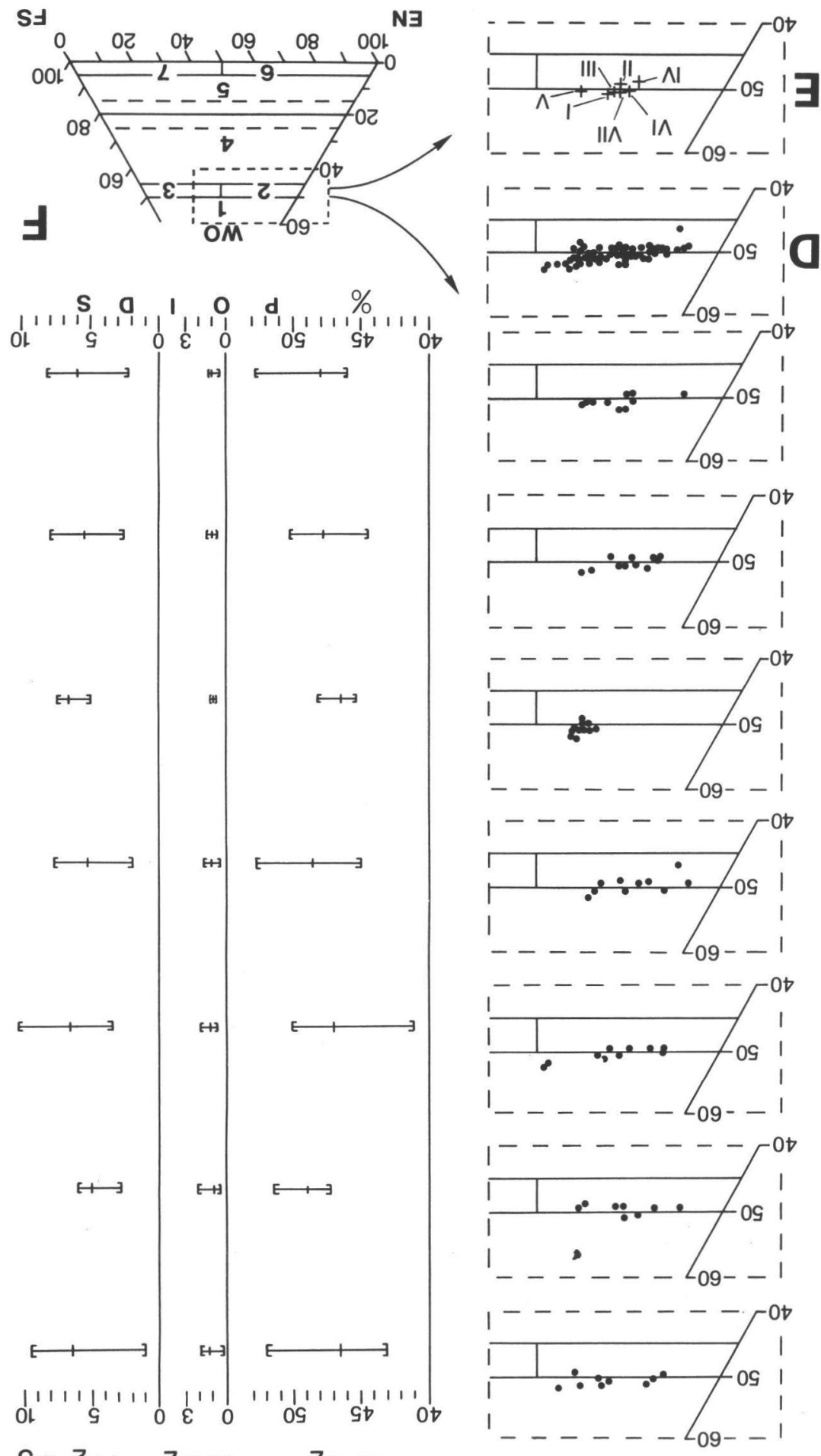

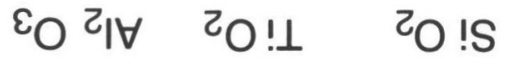
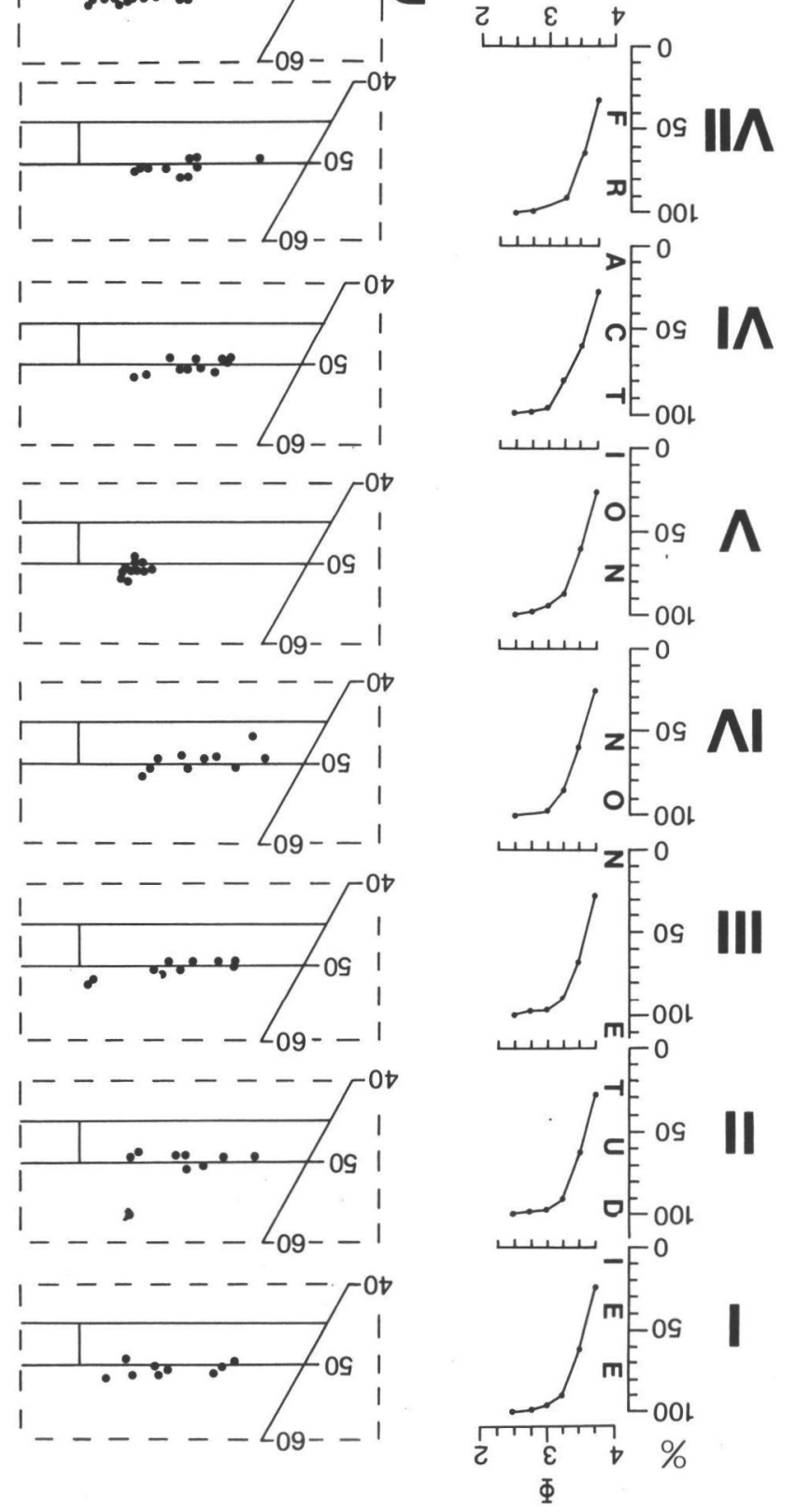

0

$\mathbf{g}$ 
argilisé en place et du loess a pénétré les couches de téphra. Pour éviter cette double source d'erreur, des mesures de largeur ont été réalisées au microscope sur les clinopyroxènes de plus de $74 \mu \mathrm{m}$. Cette limite inférieure a été fixée en fonction du tamisage à $63 \mu \mathrm{m}$ (voir plus haut) et tient compte de la possibilité qu'ont eue les grains de traverser les mailles du tamis suivant la diagonale.

Dans chaque gîte, $95 \%$ des grains se répartissent dans les classes plus fines que $3 \mathrm{Phi}$ et aucun grain plus gros que 2.5 Phi n'a été observé (Fig. 2A).

La petite taille des clinopyroxènes et leur distribution granulométrique sont identiques tout le long du transect étudié, soit sur environ $250 \mathrm{~km}$ de distance. En comparant avec les données granulométriques relatives à la retombée de l'éruption récente du Mont St. Helens (SARNA-WOJCICKI et al. 1981), tous les gîtes étudiés devraient être éloignés de plusieurs centaines de kilomètres du lieu d'émission.

\section{Composition chimique globale de la téphra}

Dans les différents gîtes, le verre de la téphra est à ce point altéré qu'aucune analyse d'esquilles ou de recouvrement sur les minéraux n'a donné de résultat satisfaisant. La matière d'apparence vitreuse se désintègre lors du bombardement dans la microsonde.

Comme nous avons montré plus haut que les couches de téphra sont partout contaminées par du loess, la composition chimique de la téphra a été recherchée en comparant les parts de 10 éléments majeurs de la téphra contaminée, à celles des loess purs adjacents.

\subsection{Méthode}

Des échantillons provenant d'une part de la téphra, et d'autre part des loess immédiatement sus- et sous- jacents ont été traités de la façon suivante: 1) prélèvement d'environ $50 \mathrm{mg}$ de sédiment; 2) élimination du calcaire secondaire par dissolution dans $\mathrm{HCl}$ froid et rinçages à l'eau distillée; 3) élimination des matières organiques et autres volatiles par calcination à $900^{\circ} \mathrm{C}$; 4) mélange avec une quantité égale de fondant; 5) fusion dans des creusets en platine; 6) dosages répétés par microsonde.

En conséquence de cette méthode de préparation: 1) les bouclages se font aux environs de $50 \%$, et pour chaque échantillon les résultats ont été additionnés puis normalisés à $100 \%$; 2) le fer total se trouve uniquement sous forme ferrique.

Les résultats sont présentés dans le tableau 3.

\subsection{Résultats}

Dans les colonnes $\mathrm{D}(1-7)$, nous avons déterminé par extrapolation les domaines des parts de chaque élément majeur. La tendance a été dégagée de la comparaison des valeurs propres aux loess adjacents et de celles de la téphra contaminée par ces loess. Les limites des domaines tiennent compte du fait qu'il n'est pas possible de savoir si la contamination de la téphra provient davantage des loess sus- ou sous-jacents. Les valeurs extrêmes ainsi obtenues s'appliquent à la téphra originelle, laquelle inclut nécessairement une part indéterminée de xénolithes, qui provoquent donc une déviation incontrôlable de la composition chimique du magma juvénile. Dans le raisonnement qui suit nous ne pouvons que négliger cette forme de contamination.

A partir des tendances établies pour chaque gîte, il est possible de définir des domaines communs pour six des éléments majeurs (Tab. 3: E), mais il existe des valeurs contradictoires pour $\mathrm{TiO}_{2}$ (1 cas contre 6), $\mathrm{Cr}_{2} \mathrm{O}_{3}$ (1 cas contre 6), $\mathrm{MnO}$ (2 cas contre 5), et $\mathrm{K}_{2} \mathrm{O}$ (2 cas contre 5).

Figure 2: Caractéristiques granulométriques, minéralogiques et chimiques de la couche de téphra dans chacun des sept gîtes étudiés (Les numéros I à VII correspondent à ceux de la figure 1).

A. Granulométrie des clinopyroxènes de plus de $74 \mu \mathrm{m}$.

B. Détermination des clinopyroxènes individuels de chaque gîte selon la classification de Morimoto (voir $\mathrm{F}$ ).

C. Domaines des parts de $\mathrm{SiO}_{2}, \mathrm{TiO}_{2}$ et $\mathrm{Al}_{2} \mathrm{O}_{3}$ dans les clinopyroxènes.

Les crochets délimitent les champs des valeurs, et le trait vertical correspond à la valeur moyenne.

D. Ensemble des pyroxènes des 7 gîtes.

E. Pyroxène moyen pour chacun des 7 gîtes.

F. Détermination des clinopyroxènes d'après la classification de Morimoto (1988).

Légende: WO, wollastonite; EN, enstatite; FS, ferrosilite. -

1 , pyroxènes inhabituels; $2-3$, série diopside-hédenbergite; 4 , augite;

5, pigeonite; $6-7$, série enstatite-ferrosilite. 
Tableau 3: Composition chimique d'échantillons de roches fondues provenant de la couche de téphra et des loess immédiatement sus- et sous-jacents de chacun des gîtes étudiés.

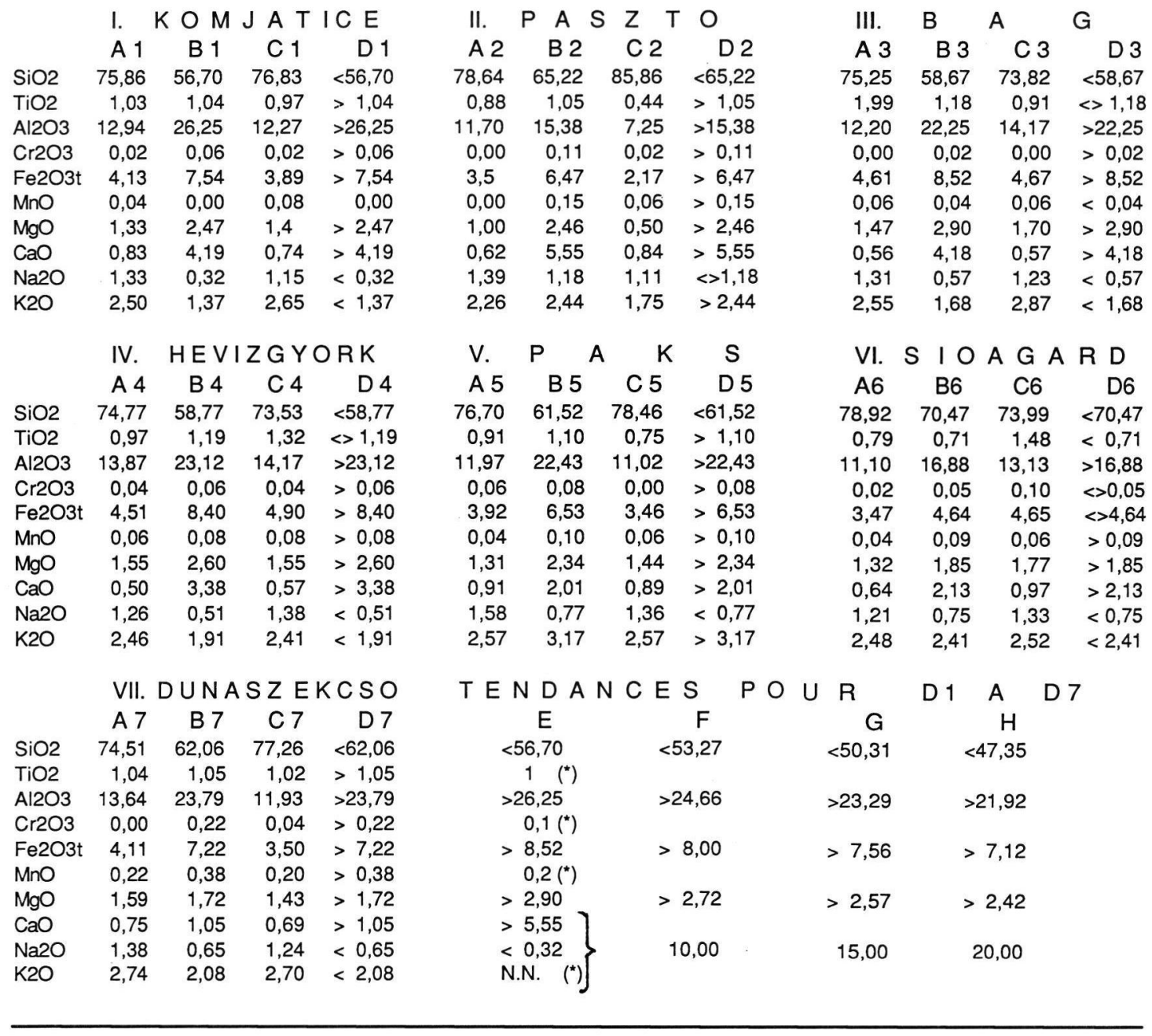

Légende: A $1-7$, loess sus-jacent; $B 1-7$, téphra brute (donc lessivée et contaminée par les loess adjacents); $C 1-7$, loess sous-jacent; D1-7, domaines comprenant les parts d'éléments majeurs de la téphra (donc sans contamination loessique, mais avec ses xénolithes); $\mathrm{E}$, domaines communs des valeurs $\mathrm{D} 1$ à $\mathrm{D} 7$. Afin de pouvoir poursuivre le calcul, les valeurs marquées $(*)$ ont été fixées arbitrairement en fonction de l'existence des données contradictoires. N.N. = valeur non nécessaire pour pousuivre le calcul; $\mathrm{F}, \mathrm{G}, \mathrm{H}$, valeurs de la colonne $\mathrm{E}$ recalculées en fixant $\mathrm{CaO}+\mathrm{Na}_{2} \mathrm{O}+\mathrm{K}_{2} \mathrm{O}$ respectivement à $10 \%, 15 \%$ et $20 \%$.

Les valeurs contradictoires précitées affectent des éléments peu abondants: $\mathrm{TiO}_{2}<1,99 \%, \mathrm{Cr}_{2} \mathrm{O}_{3}<$ $0,22 \%, \mathrm{MnO}<0,38 \%$ et $\mathrm{Na}_{2} \mathrm{O}<1,58 \%$. Pour $\mathrm{Cr}$ et $\mathrm{Mn}$, les valeurs mesurées $(=1 / 2$ des valeurs exprimées: revoir méthode de préparation) se situent près des limites de détection et peuvent être considérées comme non significatives.
L'existence des valeurs contradictoires peut aussi s'expliquer par le lessivage du verre désagrégé. Ce processus est bien connu dans la palagonitisation au cours de laquelle des éléments mobiles sont emportés, provoquant un enrichissement passif en éléments immobiles (v. FISHER \& SCHMINCKE 1984). 
Dans une recherche identique réalisée dans une coupe de loess d'Allemagne moyenne, Juvigné \& SeidenSCHWANN (1989) ont montré que le lessivage affectait particulièrement $\mathrm{CaO}, \mathrm{Na}_{2} \mathrm{O}$ et $\mathrm{K}_{2} \mathrm{O}$, sans que l'on puisse quantifier l'effet sur chaque élément particulier.

Dans le cas présent, seule la part anormalement faible de $\mathrm{Na}_{2} \mathrm{O}(<0.32 \%)$ dans la téphra étudiée, atteste l'influence du lessivage du verre. La comparaison des parts cumulées de $\mathrm{CaO}+\mathrm{Na}_{2} \mathrm{O}+\mathrm{K}_{2} \mathrm{O}$ de la téphra, avec celles des roches volcaniques fraîches ne peut être utilisée pour juger du lessivage, dans la mesure où on ne connait que des valeurs minimums ou maximums de ces éléments dans la téphra. Quant à la sous-représentation de $\mathrm{Na}_{2} \mathrm{O}$ par rapport à $\mathrm{K}_{2} \mathrm{O}$, elle n'est pas non plus déterminante dans la mesure où des rapports $\mathrm{Na}_{2} \mathrm{O} / \mathrm{K}_{2} \mathrm{O}<1$ existent notamment dans les leucitites (IRVINE \& BARAGAR 1971; VIERECK 1984).

Pour compenser l'effet du lessivage, les valeurs de la colonne $\mathrm{E}$ ont été recalculées en fixant $\mathrm{CaO}+\mathrm{Na}_{2} \mathrm{O}$ $+\mathrm{K}_{2} \mathrm{O}$ égal à $10 \%, 15 \%$ et $20 \%$ (Tab. $3: \mathrm{F}, \mathrm{G}, \mathrm{H}$ ). Pour permettre ce calcul, les parts contradictoires de $\mathrm{TiO}_{2}, \mathrm{Cr}_{2} \mathrm{O}_{3}$ et $\mathrm{MnO}$ ont été fixées respectivement à $1,0.1$ et 0.2 .

Remarque. - Les valeurs $10 \%, 15 \%$ et $20 \%$ tiennent compte du fait que dans la plupart des roches volcaniques, les parts cumulées de $\mathrm{CaO}+\mathrm{Na}_{2} \mathrm{O}$ $+\mathrm{K}_{2} \mathrm{O}$ sont généralement comprises entre 10 et $15 \%$; ce n'est que dans les roches nettement sous-saturées (néphélinite, leucitite ...) que les valeurs se situent généralement entre 15 et $20 \%$ (IRVINE \& BARAGAR 1971; VIERECK 1984).

Les valeurs obtenues par le calcul décrit ci-dessus permettent de mieux cerner les parts de 4 éléments bien représentés dans la téphra: $\mathrm{SiO}_{2}, \mathrm{Al}_{2} \mathrm{O}_{3}, \mathrm{Fe}_{2} \mathrm{O}_{3}$ et $\mathrm{MgO}$. Les tendances qui se dégagent des valeurs des colonnes $\mathrm{F}, \mathrm{G}$ et $\mathrm{H}$ du tableau 3 indiquent que la téphra doit être basique ou à peine intermédiaire $\left(\mathrm{SiO}_{2}<53 \%\right)$, riche en fer $(>7 \%)$, et exceptionellement riche en alumine $(>22 \%)$; elle est plutôt pauvre en magnésium $(>2.4 \%)$, ce qui atteste l'état nettement différencié de son magma.

\section{Unicité de la retombée}

L'association de minéraux mafiques est partout dominée par un clinopyroxène très calcique, alumineux et peut-être ferrique.

La distribution granulométrique des clinopyroxènes tout le long du transect ne montre aucune variation qui permette de distinguer plusieurs retombées.
Pour les éléments majeurs les plus abondants, il existe des domaines de valeurs communs à la téphra des sept gîtes étudiés. Les erreurs de mesures à la microsonde et le lessivage du verre in situ expliquent, au moins partiellement, quelques valeurs contradictoires propres au titane, au chrome, au potassium et au manganèse.

La couleur de la téphra peut être grise ou jaune-brun suivant les gîtes; toutefois, cette différence n'est pas déterminante pour distinguer plusieurs retombées, dans la mesure où elle peut être le résultat de l'altération différentielle subie in situ.

En conséquence, nous pensons que la téphra étudiée est le résultat d'une retombée unique; nous l'appellerons "Téphra de Bag", car dans cette localité, la coupe est aisément accessible et n'est pas menacée de disparition.

Remarque. - Au cours de nos recherches, nous n'avons pas trouvé de téphra à amphibole et biotite telle que celle qui a été décrite par KRIVAN (1958) et KRIVAN \& ROZASVOLGYI (1964). D'après les descriptions de ces auteurs, la position stratigraphique est très proche de celle de la téphra de Komjatice. La question se pose donc de savoir s'il existe ou non deux retombées pratiquement synchrones dans les loess de Hongrie.

\section{Age de la téphra}

Le stratotype de Paks est particulièrement intéressant pour discuter l'âge absolu de la retombée. La couche de téphra s'y trouve entre les sols BA et MB (Fig. 1B). Diverses interprétations chronostratigraphiques de cette coupe ont été proposées. Il en résulte les possibilités suivantes pour l'âge de la téphra:

- pendant le dépôt du loess du Würm inférieur (PeCSI 1979),

— entre $87.000 \mathrm{BP}$ et $98.000 \mathrm{BP}$ d'après les datations par thermoluminescence réalisées sur des loess de Hongrie par BUTRYM \& MARUSZCZAK (1984),

- pendant le dépôt du loess Riss I vers 200.000 BP selon KRIVAN (1958).

- au début du Riss I situé vers 400.000 BP selon VASKOVSKY (1977).

- un peu moins de 300.000 ans selon HAHN (1989) qui rapporte le sol MB (Fig. 1) à l'interglaciaire Mindel/Riss, et lui attribue un âge d'environ 300.000 ans sur la base de la vitesse de sédimentation.

- nettement antérieur au dernier interglaciaire d'après la datation par thermoluminescence du 
loess du sol MF2 (WINTLE \& PACKMAN 1988). Pour ces auteurs le sol MF2 devrait être eemien, et il faut remarquer que trois autres reliques de sols interglaciaires (BD1, BD2 et $\mathrm{BA}$ ) se trouvent entre le sol MF2 et la téphra.

- près de la transition entre les cycles glaciaires $\mathrm{E}$ et $\mathrm{F}$ sur la base de la stratigraphie de KUKLA (1977). Cette transition est datée d'environ $400.000 \mathrm{BP}$.

En conclusion, les données chronostratigraphiques obtenues par l'étude des carottes océaniques (SHAKLETON \& OPDYKE 1976), et appliquées aux interprétations précitées, laissent la possibilité de situer la téphra de la coupe de Paks entre environ 60.000 BP et 400.000 BP.

Pour notre part, nous considérons que les sols fossiles qui surmontent la téphra dans les coupes étudiées sont tellement évolués qu'ils doivent être rapportés à des périodes interglaciaires. L'hypothèse d'une retombée volcanique unique que nous défendons, implique que des sols manquent dans certaines coupes en raison de l'érosion qu'elles ont subie ou de la surimpression de sols diachroniques. Les quatre sols fossiles observés postérieurement à la retombée constituent donc un nombre minimum. En conclusion, nous pensons que la retombée volcanique a eu lieu pendant ou avant la

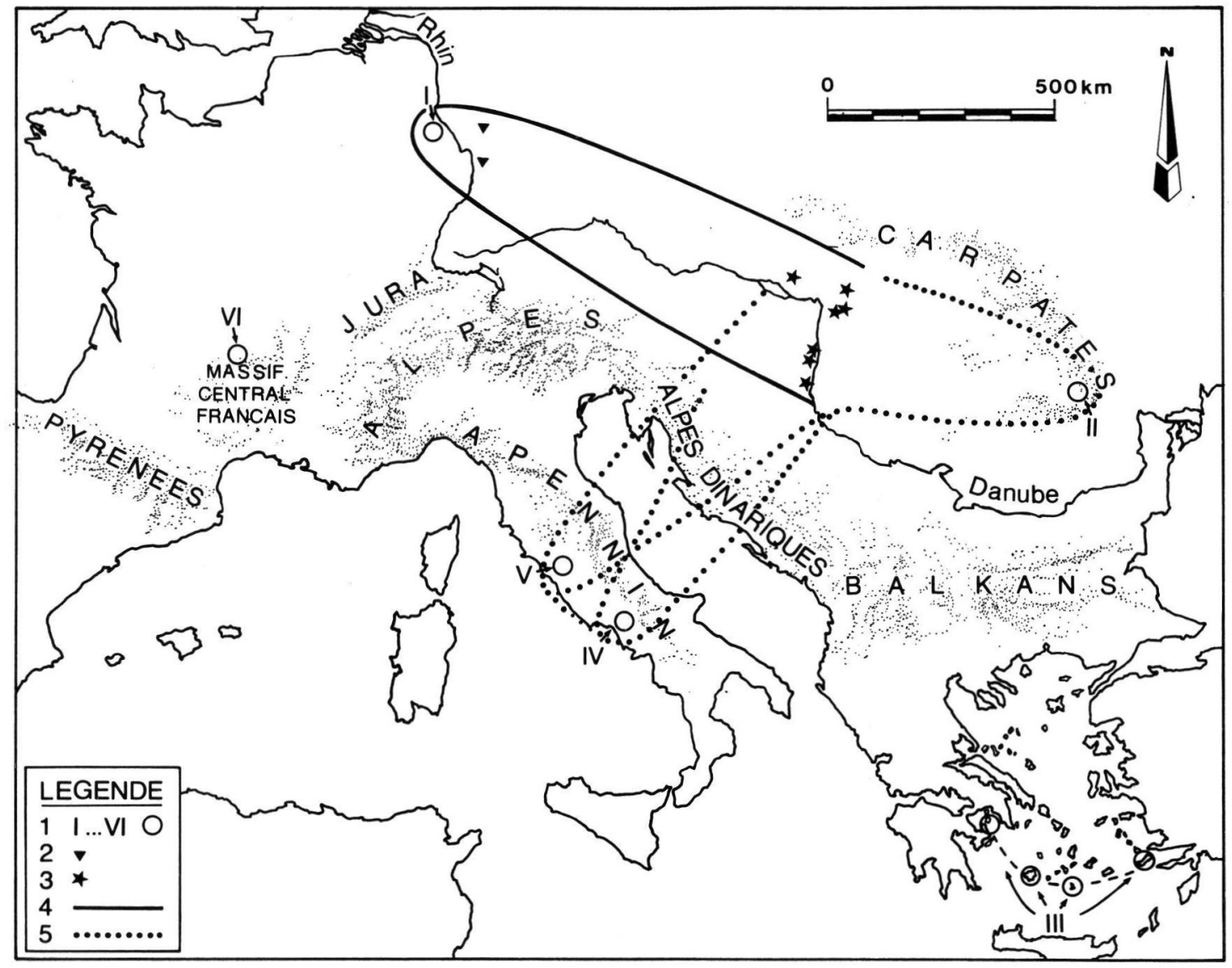

Figure 3: Les champs volcaniques quaternaires les plus proches des gîtes étudiés.

Légende: -1 , régions à forte activité explosive connue au Pléistocène inférieur à moyen:

I, Eifel orientale; II, Carpates orientales; III, arc de la Mer Egée; IV, province campanienne;

$\mathrm{V}$, province romaine; VI, Massif Central français.

-2 , occurrences distales de téphras émises par des volcans de l'Eifel orientale au cours du Pléistocène inférieur à moyen. -3 , gîtes étudiés.

-4 , esquisse du lobe le plus probable.

-5 , esquisse de lobes possibles. 
cinquième dernière glaciation, soit avant 380.000 ans selon l'échelle de SHACKLETON \& OPDYKE (1976). Toutefois, l'âge maximum acceptable est nécessairement plus récent que celui de la transition Matuyama/ Brunhes ( 730.000 ans), car le paléomagnétisme de tout le profil de Paks est positif (HaHN 1989).

\section{Origine de la téphra}

La petite taille des clinopyroxènes et leur distribution granulométrique régulière tout le long du transect étudié attestent que la Téphra de Bag provient d'un volcan éloigné de plusieurs centaines de kilomètres.

Les champs volcaniques les plus proches qui ont eu une activité fortement explosive pendant le Pléistocène sont à des distances de l'ordre de 550 à $1300 \mathrm{~km}$ (Fig. 3): les Carpates orientales, la Péninsule italienne, l'Eifel, le Massif Central français et la Grèce. La très large distribution des téphras de quelques grandes explosions historiques montre qu'aucune des distances ci-dessus ne constitue un obstacle à la corrélation.

Toutefois, aucune couche de téphra n'a été signalée, à espace régulier, entre la région étudiée et un des champs volcaniques précités (Fig. 3). Cette situation est paradoxale puisque la téphra doit exister en plus forte épaisseur et en faciès plus grossier dans des loess d'au moins une des régions suivantes: Hongrie orientale, Transylvanie, Yougoslavie, Autriche, ou Allemagne méridionale. Cette lacune exclut que l'on puisse utiliser la méthode d'observation de proche en proche qui est de loin la plus fiable pour rapporter une téphra à un volcan.

La discussion sur les possibilités de corrélation ne peut être basée que sur les données minéralogiques et géochimiques connues d'une part pour la téphra, et d'autre part pour chacun des champs volcaniques environnants. Une discussion exhaustive de ce problème serait extrêmement vaste et purement conjecturale. D'une part, le type de magma juvénile de la Téphra de Bag n'est pas connu avec précision, alors que ses pyroxènes sont maintenant bien connus; d'autre part tous les magmas des champs volcaniques environnants n'ont pas été déterminés, les positions stratigraphiques de leurs phases explosives ne sont que partiellement connues et leurs pyroxènes n'ont été que très exception ellement déterminés sur la base de la composition chimique.

En conséquence, la recherche de l'origine de la téphra se résumera ici à évoquer quelques grandes éruptions explosives du Pléistocène moyen à inférieur dans les champs volcaniques environnants.

\subsection{Carpates orientales (Roumanie)}

Le champ volcanique néogène des Carpates orientales (Monts Calimani, Gurghiu et Harghita) est le plus proche des gîtes étudiés $(550 \mathrm{~km})$. Toutefois, les dernières éruptions connues à ce jour datent d'environ 1.450 .000 ans (PELTZ et al. 1987), mais quelques auteurs soupçonnent l'existence de manifestations plus récentes (RADULESCU 1973).

Les produits de ce champ volcanique sont essentiellement andésitiques et comprennent plusieurs générations d'andésites à pyroxènes (PELTZ et al. 1973). Dans ces derniers magmas, les parts cumulées de $\mathrm{CaO}+\mathrm{Na}_{2} \mathrm{O}+\mathrm{K}_{2} \mathrm{O}$ sont toujours comprises entre 10 et $15 \%$, et la part la plus élevée connue de $\mathrm{Al}_{2} \mathrm{O}_{3}$ est de $22,96 \%$ (PELTZ et al. 1973). Si on fixe arbitrairement à $15 \%$ la teneur en $\mathrm{CaO}+\mathrm{Na}_{2} \mathrm{O}+\mathrm{K}_{2} \mathrm{O}$ dans la Téphra de $\mathrm{Bag}$, la part de $\mathrm{Al}_{2} \mathrm{O}_{3}$ de cette téphra devient $23,29 \%$ (Tab. 3, col. G), ce qui est supérieur à la teneur maximum connue dans les andésites à pyroxènes des Carpates.

L'origine carpatique de la téphra de Bag est donc compromise aussi bien par les données chronostratigraphiques que géochimiques.

\subsection{Eifel orientale (Allemagne)}

Plusieurs couches de téphras ont été observées dans des loess antérieurs au Pléistocène supérieur en Allemagne moyenne à plus de $100 \mathrm{~km}$ à l'Est et au SudEst de l'Eifel orientale d'où elles proviennent (BIBUS 1973, 1974, 1976, 1980; SEIDENSCHWANN \& JUVIGNÉ 1986; JUVIGNÉ \& SEIDENSCHWANN 1989). Ces occurrences sont situées sur la trajectoire possible de nuages de téphras qui se seraient déplacés de l'Eifel vers l'Europe centrale (Fig. 3).

Les magmas différenciés émis pendant le Quaternaire dans l'Eifel orientale sont basiques à intermédiaires (SIMON 1969; FRECHEN 1976; VIERECK 1984; SCHMINCKE et al. 1983). De plus, certains d'entr'eux contiennent de 20 à $24 \%$ d'alumine; comme leurs parts cumulées de $\mathrm{CaO}+\mathrm{Na}_{2} \mathrm{O}+\mathrm{K}_{2} \mathrm{O}$ peuvent atteindre $20 \%$, la comparaison des valeurs de la Téphra de Bag doit se faire avec les valeurs des colonnes $\mathrm{G}$ et $\mathrm{H}$ du tableau 3.

Les puissants tufs de Rieden (VIERECK 1984) qui ont été datés récemment d'environ 410.000 à $465.000 \mathrm{BP}$ (BOGAARD et al. 1987; BOGAARD \& SCHMINCKE 1988) méritent une citation particulière dans cette discussion, même si les plus riches en alumine $\left(\mathrm{Al}_{2} \mathrm{O}_{3}>\right.$ $20 \%$ ) sont un peu plus pauvres en $\mathrm{CaO}$ et en $\mathrm{MgO}$ que la Téphra de Bag. 
Les cinq téphras découvertes récemment près de Frankfurt a.M./Allemagne (JUVIGNE \& SEIDENSCHWANN 1989) doivent aussi être mises en exergue dans la tentative de corrélation de la Téphra de Bag. Elles ont été émises dans l'Eifel orientale, et sont situées dans des loess de périodes froides du complexe cromérien (sensu ZAGWIJN 1985). Leurs clinopyroxènes sont toujours alumineux et fortement calciques. Parmi les cinq retombées, la téphra ALZ-2 est aussi particulièrement riche en $\mathrm{Al}_{2} \mathrm{O}_{3}$.

En conséquence, dans l'état actuel des connaissances, il n'existe pas d'incompatibilité fondamentale entre la composition de la série de magmas quaternaires de l'Eifel orientale, et celle de la Téphra de Bag.

\subsection{Monts Dore (Massif Central français)}

Des éruptions violemment explosives ont eu lieu dans les Monts Dore pendant le Pléistocène moyen à inférieur, mais les magmas correspondants appartiennent pour l'essentiel, à une série saturée (CANTAGREL \& BAUBRON 1983). Cette dernière caractéristique est incompatible avec la faible teneur en silice de la Téphra de Bag.

\subsection{Apennin central (Italie)}

D'importantes émissions de téphras sont connues dans cette région pendant le Pléistocène moyen à inférieur (voir PICHLER $1970 \mathrm{a}, \mathrm{b}$ ). Celles de la province toscane sont à exclure de la corrélation car elles appartiennent essentiellement à une série saturée, mais celles des provinces romaine (Mont Sabatini) et campanienne (Roccamonfina) se classent dans des séries sous-saturées. Toutefois, des parts d'alumine supérieure à $20 \%$ n'ont été rapportées dans aucune des téphras.

\subsection{Arc de la Mer Egée (Grèce)}

Des éruptions ont eu lieu à différents moments du Pléistocène dans l'arc volcanique de la Mer Egée (FYTIKAS et al. 1984). Les produits émis appartiennent à la série calco-alcaline (andésites basaltiques, andésites, dacites, rhyolites). En général, les teneurs en $\mathrm{SiO}_{2}$ de ces roches $(54$ à $73 \%$ ) sont nettement supérieures aux valeurs probables de la Téphra de Bag $(<53 \%)$. Inversement la part de $\mathrm{Al}_{2} \mathrm{O}_{3}$ varie entre 12 et $18 \%$ dans les magmas pléistocènes de la mer Egée, et est supérieure à $21 \%$ dans la Téphra de Bag. On peut donc écarter la possibilité que la Téphra de Bag provienne d'un volcan de la Mer Egée.

\subsection{Synthèse}

En ce qui concerne l'origine géographique de la Téphra de Bag, les données actuellement disponibles soutiennent en priorité la corrélation avec l'Eifel orientale (Fig. 3). Toutefois, deux provinces volcaniques de l'Apennin central et une des Carpates orientales ne peuvent être formellement exclues comme lieux d'origine (Fig. 3).

\section{Conclusion}

Il existe en Europe centrale une téphra appartenant à une retombée volcanique à très large dispersion survenue probablement pendant ou avant la cinquième dernière glaciation, soit avant $380.000 \mathrm{BP}$, mais après l'inversion magnétique Matuyama/Brunhes. Elle est caractérisée par un magma basique à intermédiaire, très riche en alumine, et son minéral mafique essentiel est un clinopyroxène alumineux et très calcique.

Cette téphra permet d'établir de nouvelles corrélations entre les coupes de loess concernées de Tchécoslovaquie et de Hongrie. Elle met en évidence des lacunes stratigraphiques qui se manifestent notamment par l'absence, dans certaines coupes, de un ou plusieurs paléosol(s) interglaciaire(s) du Pléistocène moyen ou supérieur.

L'origine de cette retombée ne peut encore être déterminée avec certitude dans la mesure où la téphra n'a pas encore été découverte, à espaces réguliers, entre le transect étudié et un des champs volcaniques quaternaires d'Europe. Les compositions chimiques du magma et des pyroxènes de la Téphra de Bag sont compatibles avec celles de téphras du Pléistocène inférieur à moyen de l'Eifel orientale. Des ressemblances identiques n'ont pu être mises en évidence avec aucun autre champ volcanique pléistocène d'Europe.

\section{Remerciements:}

Nous remercions vivement Mr. J. WAUTIER qui a réalisé les analyses chimiques au Centre d'Analyse par Microsonde pour les Sciences de la Terre, à l'Université Catholique de Louvain (Belgique). Cette recherche a été réalisée dans le cadre des Accords Culturels entre les Université de Liège et Eötvös Loránd de Budapest. Toutes les personnes qui contribuent à mener à bien ces échanges reçoivent aussi nos vifs remerciements. 


\section{Références}

BiBUS, E. (1973): Ausbildung und Lagerungsverhältnisse quartärer Tuffvorkommen in der Wetterau. - Notizbl. hess. L.-Amt Bodenforsch., 101: 346-361; Wiesbaden.

- (1974): Das Quartärprofil im Braunkohletagebau Heuchelheim (Wetterau) und seine vulkanischen Einschaltungen. - Notizbl. Hess. L.-Amt Bodenforsch., 102: 159-167; Wiesbaden.

- (1976): Pliozän und Quartär der Wetterau und ihrer Randgebiete. - Rhein-Main Forsch., 82: 17-42; Frankfurt a. M.

- (1980): Zur Relief-, Boden- und Sedimententwicklung am unteren Mittelrhein. - Frankfurter geow. Arb., Serie D, Bd. 1: 296 S.; Frankfurt a. M.

BogaARd, P. v. D., HALL, C. M., SCHMinCKe, H.-U. \& YoRK, D. (1987): ${ }^{40} \mathrm{Ar} / 39 \mathrm{Ar}$ Laser dating of single grains: ages of Quaternary tephra from the East Eifel volcanic field, FRG. - Geophysical Research Letter, 14/12: 1211-1214; Washington.

— \& SCHMINCKE, H.-U. (1988): Aschenlagen als quartäre Zeitmarken in Mitteleuropa. - Die Geowiss., 6: 7584; Bonn.

ButRYM, J. \& MURUSZCAK, H. (1984): Thermoluminescence chronology of younger and older loesses. - In PÉCSI, M. (ed.): Lithology and Stratigraphy of Loess and Paleosoils, p. 195-199; Budapest (Hungarian Academy of Sciences).

CAntagrel, J.-M. \& Baubron, J.-C. (1983): Chronologie des éruptions dans le massif volcanique des Monts Dore (Méthode potassium-argon), Implications volcanologiques. - Géologie de la France, (2), n'1 -2: 123-142; Orléans (BRGM).

FiSHER, R. V. \& SCHMINCKE, H.-U. (1984): Pyroclastic rocks. -472 p.; Berlin (Springer).

FRECHEN, J. (1976): Siebengebirge am Rhein, Laacher Vulkangebiet, Maargebiet der Westeifel, Vulkanologischpetrographische Exkursionen. - Sammlung Geologischer Führer, 56, 3e édition: 209 p.; Berlin, Stuttgart (Gebr. Bornträger).

Fytikas, M., InNocenti, F., Manetti, P., Mazzuoli, R., PecCerillo, A. \& Villari, L. (1984): Tertiary to Quaternary evolution of volcanism in the Aegean region. - In: The geological evolution of volcanism of the Eastern Mediterranean. Sp. Publ. Geol. Soc., 17: Dixon, J. E. \& ROBERTSON, A. H. F., eds.: 687-699; Oxford, London.

HAHN GY. (1989): A magyarországi löszök kronosztratigráfiája. - Thèse Doct, Acad. hongroise des Sciences; 20 p.; Budapest. - [Manuscrit].

Hamm M.-H. \& Vieten, K. (1971): Zur Berechnung der kristallchemischen Formel und des $\mathrm{Fe}^{3+}$-Gehaltes von Klinopyroxenen aus Elektronenstrahl-Mikroanalysen. N. Jb. Miner. Mh., 1971: 310-314; Stuttgart.
IRVINE, T. \& BARAGAR, W. (1971): A guide to the chemical classification of the common volcanic rocks. - Canadian Journal of Earth Sciences, 8: 523-548; Ottawa.

Juvigné, E. \& Seidenschwann, G. (1989): Das Talverschüttungsprofil von Alzenau i. Ufr. (Ziegeleigrube Zeller) eine Typlokalitat früh-mittelpleistozäner Tephren. - Jber. wetterau. Ges. ges. Naturkunde, 140-141. Jg.: 143-172; Hanau.

KRIVAN, P. (1958): Traces du volcanisme andésitique pléistocène supérieur (rissien) de la zone des Carpates dans le profil de loess fondamental de Paks. - Ann. Univ. Scient. Bud. Sect. Geol., Tom. II: 99-105; Budapest.

— \& Rozsavolgyi, J. (1964): Andezittufit vezetöszint a magyarországi felsöpleisztocén (Rissi) lösz-szelvényekböl. - Földtani Közlöny (Bull. Soc. Géol. Hongrie), 94: 257-265; Budapest.

KuKLA, G. (1977): Pleistocene Land-Sea correlations 1. Europe. - Earth Science Reviews, 13: 307-374; Amsterdam.

Morimoto, N. (1988): Nomenclature of pyroxenes- Bull. Minéral., 111: 535-550; Paris.

PÉCSI, M. (1979): Lithostratigraphical subdivision of the loess profiles at Paks. - Acta Geologica Scientarium Hungaricae, 22: 409-418; Budapest.

Peltz, S., Vajdea, E., Balogh, K. \& Pecskay, Z. (1987): Contributions to the chronological study of the volcanic processes in the Calimani and Harghita Mountains (East Carpathians, Romania). - D. S. Inst. Geofiz., 72-73/1: 323-338; Bucarest.

- Vasiliu, C., Udrescu, C. \& VASIlescu, A. (1973): Geochemistry of volcanic rocks from the Calimani, Gurghiu, and Harghita Mountains (Major and trace elements). In: Vulcanismul Neogen al Lantului Muntos CalimaniGurghiu-Harghita, Simpozionului International de Vulcanologie, 42: 339-393; Bucarest.

PICHLER, H. (1970a): Italienische Vulkan-Gebiete I: SommaVesuv, Latium, Toscana. - Sammlung Geologischer Führer, 51: 258 p.; Berlin, Stuttgart (Gebr. Borntraeger).

- (1970b): Italienische Vulkan-Gebiete II: Phlegraische Felder, Ischia, Ponza-Inseln. Roccamonfina. - Sammlung Geologischer Führer, 52, 186 p.; Berlin, Stuttgart (Gebr. Borntraeger).

Radulescu, D. P. (1973): Position of the Calimani-Gurghiu-Harghita area within the Neozoic volcanic zone of the alpine regions. - In: Vulcanismul Neogen al Lantului Muntos Calimani-Gurghiu-Harghita. Simpozionului International de Vulcanologie. Anuarul Institutului Geologic, 41: 7-15; Bucarest.

Sarna-Wojcicki, A., Shipley, S., Waitt, R., Dzurisin, D. \& WoOD, S. (1981): Areal distribution, thickness, mass, volume, and grain-size of air-fall ash from the six major eruptions of 1980. - In: The 1980 eruptions of Mount St. Helens, Washington, Lippman, P. \& Mullineaux, ed., U.S.A. Geological Survey Professional Paper, 1250: 577-600; Denver/Colorado. 
SHAKIETON, N. \& OPDYKe, N. (1976): Oxygen isotope and paleomagnetic stratigraphy of equatorial Pacific core V8239, Late Pliocene to latest Pleistocene. - In: Investigation of Late Quaternary Paleoceanography and Paleoclimatology, es. R. M. CLINE \& J. D. HAYS: Geol. Soc. Amer. Memoir, 145: 449-464; Boulder/Co.

SCHMinCKE, H.-U., LORENZ, V. \& SeCK, H. A. (1983): The Quarternary Eifel volcanic fields. - In: Plateau Uplift, ed. FucHs et al.; 139-151; Berlin, Heidelberg (Springer).

SeidenschwanN, G. \& JuvignE, E. (1986): Fundstellen mittelpleistozäner Tephralagen im Bereich des kristallinen Vorspessarts. Stratigraphie quartärer Tephren. Z. dt. Geol. Ges., 137: 613-623; Hannover.

SIMON, R. (1969): Geochemische und petrographische Untersuchungen an den Laacher Trachyten und Olivintrachyten sowie an den Laacher-See-Tuffen. - Disser- tation, Universität zu Köln, Mathematisch-Naturwissenschaftliche Fakultät; 135 S., Köln.

VASKOWSKY, I. (1977): Kvarter Slovenska (Quaternary of Slovakia). - 247 p.; Bratislava. - [Engl. summary].

— \& Karolusova, E. (1969): Prvy nalez vulkanickeho popola v sprasiach Komjaticej tehelne. - Geol. Prace, Geol. Ust. D. Stura, 50; Bratislava.

VIERECK, L. (1984): Geologische und petrologische Entwicklung des pleistozänen Vulkankomplexes Rieden, Ost-Eifel. - Bochumer geol. u. geotechn. Arb., 17: 337 p.; Bochum.

Wintle, A. \& PACKMan, S. (1988): Thermoluminescence ages for three sections in Hungary. - Quaternary Science Reviews, 7: 315-320; London.

ZAGWIJN, W. H. (1985): An outline of the Quaternary stratigraphy of the Netherlands. - Geol. Mijnbouw, 64: 17-24; Dordrecht. 